\title{
Quantitative evaluation of aging in bearings and electric brushes using infrared thermography
}

\author{
by A. MADRID (*)
}

(*) Engineering Consultant, Ramonet 48/50, Madrid 28033, Spain.

\begin{abstract}
The quantitative evaluation of aging in both bearings and electric brushes, and the estimation of their respective failure rates by means of IR thermal mapping are discussed using the DIRT-FREE technique. Aging is characterized by component wear due to friction-induced dissipative heating and mild corrosion. Ad hoc thermal analyses yield both the actual component wears and wear rates, and the basis for the appraisal of potential thermal stresses in regard to component failure.
\end{abstract}

\section{Introduction}

A quantitative method based on conventional IR thermographic measurements - the Direct IR Thermography Failure Rate Energetic Evaluation (DIRT-FREE) technique - has been recently proposed for both yielding an accurate diagnosis of hardware degradation and/or failure, and estimating realistic values of their respective failure rates as applied to aging and secondary failures [1-2]. Hardware diagnosis is accomplished by means of ad hoc thermal analyses based on the inverse heat-transfer problem corresponding to the relevant thermal patterns actually mapped by means of IR thermography. Then, the load-strength interference theory of reliability is subsequently used as appropriate to yield the failure rate estimates. The DIRT-FREE technique is applicable to components being in continuous operation, or on standby, that are over each time interval of interest: 1) exposed to sensibly-steady normal working conditions and undergoing natural aging; or, 2) either subjected to rather steady abnormal circumstances or bearing a quasi-steady permanent damage, caused by past out-of-tolerance conditions, that may alter the actual failure rates of some failure modes which are possible under normal working conditions. If the actual working conditions or damages are otherwise markedly time-dependent the DIRT-FREE technique may be applicable also [2]. Then, this research concentrates on the quantitative evaluation of aging in bearings and electric brushes, as basic components of mechanical and electrical heavy rotating machines, due mainly to friction-induced dissipative heating and possibly to mild corrosion by means of the DIRT-FREE technique. The results obtained should be useful and readily applicable if decision making about specific corrective measures is dictated as usual by arriving quickly and early enough at an adequate resolution. An efficient implementation of the DIRT-FREE technique would usually demand high-resolution IR equipment measurements and dedicated software availability also.

\section{Bearing/electric brush aging degradation analysis}

The actual degradation status due to aging in both bearings and electric brushes may be rightly characterized and quantitatively evaluated by means of the corresponding wears present in the respective components. This is the approach adopted in this work as follows. Then, regardless of the actual deterioration mechanisms, each specific physical situation in the above components may be represented at any time interval of interest by way of a set of independent systems of equations in the quantities playing a relevant role on the referred inverse heat-transfer problem. The solution of the latter systems may be uniquely obtained provided that definite accessible quantities are concurrently measured by suitable means which, in turn, yields as diagnostic result the actual component wears as shown below. In doing so, both typical friction bearings and electric brushes are modelled 
according to reasonable idealizations that allow meaningful and simplified analyses of the actual aging process, and can be easily extended to other relevant situations if desired.

\subsection{Friction bearing wear diagnosis}

The friction bearing is modelled as two vertical coaxial cylindrical bodies of like material and infinite extent, and a narrow sealed annulus between them of thickness $\delta$ filled up with a lubricating fluid as shown in figure 1 . This fluid is regarded as being incompressible, Newtonian and of uniform viscosity $\mu$ at all times. The inner and outer cylinders are subject to a sensibly-steady relative angular velocity $\Omega$. Quasi-steady state laminar flow is postulated for the lubricating fluid. Quasi-steady state heat conduction is assumed to be present in the bearing in such way that axial heat conduction is negligible, and heat flows essentially with radial symmetry. The thermal conductivity of the outer hollow cylinder $k$ is presumed to be constant over each time interval of interest, as well as $h_{a}$ that stands for the addition of both the convective and linear radiative heat-transfer coefficients between the bearing external surface and the ambient. The bearing wear is thought to be caused by mild corrosion and/or friction-induced erosion which are modelled as taking place exclusively at either of its annular surfaces, assuming that the corrosion and/or erosion product is continuously and entirely washed away from the latter surfaces to be uniformly immersed in the lubricating fluid. Corrosion is presumed to occur slowly enough so that its inherent local heat generation may be sensibly ignored as compared to the viscous dissipative heating inside the bearing annulus at all times. The thermal gradient across the bearing annulus is assumed to be insignificant at any time also. The three last assumptions may be adequately relaxed if necessary at the expense of arriving at more complex formulations though. In particular, the incorporation of an appropriate apparent thermal conductivity $k$, and suitable deposition rates for each species may be used to account for sticking of the corrosion and/or erosion product to the bearing annular surfaces. Moreover, the hypothesis about the latter thermal gradient is otherwise usually adequate provided that $\Omega$ is not too high. Finally, figure 1 shows the radial distances $R_{i}$, $R_{O}$ and their corresponding surface temperatures $T_{i}, T_{O}$ playing a relevant role, as well as the average ambient temperature outside the thermal boundary layer $T_{a}$.

Then, the viscous dissipative heating power per unit axial length inside the bearing annulus $W_{i}$ may be calculated solving the motion equation as applied to the lubricating fluid under the above relevant assumptions [3]. In doing so, it is readily shown that $W_{i}$ may be approximated as follows:

$$
W_{i}=\frac{2 \pi \mu \Omega^{2} R_{i}^{3}}{\delta} \quad \text { if } \quad \frac{\delta}{R_{i}} \ll 1
$$

since $\delta$ is usually very small as compared to $R ;$ at all times. In other respects, a convenient relationship between $\delta$ and $T_{O}$, that is, the temperature which will be actually mapped by means of IR thermography, may be reached at solving the heat conduction equation as applied to the bearing outer hollow cylinder in conjunction with $W_{i}$ under the above relevant assumptions [3]. In doing so, it is readily obtained if $W_{i}$ is represented by (1) that:

$$
\begin{gathered}
\delta=\frac{\mu \Omega^{2} R_{i}^{3}}{\Psi} ; \quad \Psi=k\left(T_{i}-T_{o}\right)\left[\ln \left[\frac{R_{o}}{R_{i}}\right]\right]^{-1} \\
T(r)=T_{o}+\frac{R_{o} h_{a}}{k}\left(T_{o}-T_{a}\right) \ln \left(\frac{R_{o}}{r}\right) \quad ; \quad R_{i} \leq r \leq R_{o}
\end{gathered}
$$

with:

$$
\mu=\mu\left(T_{i^{\prime}}, x\right) \quad ; \quad h_{a}=\Phi\left(T_{o^{\prime}} T_{a^{\prime}} Q_{a^{\prime}} p_{a^{\prime}} \varepsilon_{o^{\prime}}, R_{o^{\prime}} L\right) \quad ; \quad k=\xi[\langle T(r)\rangle]
$$

where $T(r)$ and $\langle T(r)\rangle$ correspond to the local temperature of the outer hollow cylinder 
and its radially-weighted average respectively; $\chi$ stands for the concentration of debris in the lubricating fluid; $O_{a}$ and $\rho_{a}$ mean the average ambient gas flow rate and pressure outside the hydrodynamic boundary layer respectively; $\varepsilon_{O}$ denotes the bearing external surface emissivity; and $L$ indicates the bearing axial length. The set of relations (2) through (4) constitutes the system of equations that represents the bearing wear status at any time. It clearly shows that the actual number of physical quantities which are susceptible to being measured is insufficient to yield a unique solution of this system. However, the inaccessible quantities $\delta, R_{i}$, and $\chi$ change very slowly with time so that they may be regarded as being sensibly invariant over moderate lapses of time. This fact allows, in turn, reaching at a unique solution of the bearing wear status if three closelyspaced IR thermographic mappings of $T_{o}$ are actually performed under clearly distinct overall conditions, that is, by independently changing either one of $\Omega, T_{a}, Q_{a}$ and $p_{a}$ as being feasible, provided that $R_{O}, \varepsilon_{O}, \Omega, T_{a}, Q_{a}, p_{a}$ and $L$ (if required in the $h_{a}$ correlation) are concurrently measured along each mapping by suitable means. In doing so, a set of three independent systems of equations is obtained with each system showing similar relations as above, and sharing the latter invariant quantities though. Thus, the equality between the total number of equations and that of inaccessible quantities is achieved which yields a unique solution of this set. The latter solution contains both $\delta$ and $x$ that are used to characterize and diagnose the bearing wear status.

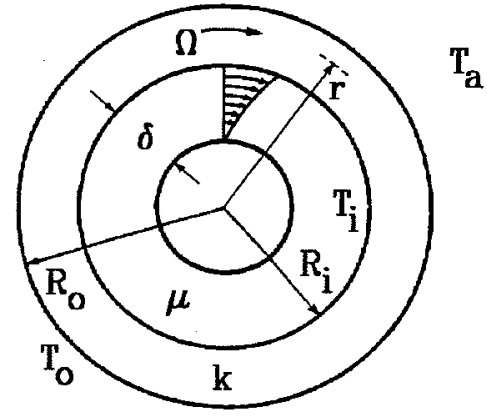

Fig. 1. -- Friction bearing cross section

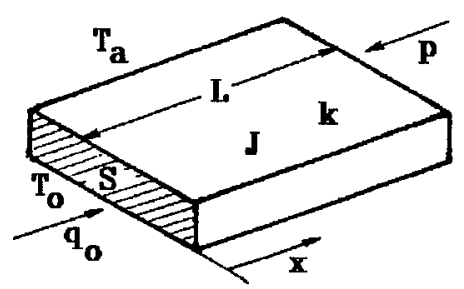

Fig. 2. -- Electric brush view

\subsection{Electric brush wear diagnosis}

The electric brush is modelled as a thin and straight conductive fin of uniform thickness and length $L$ that drives a sensibly-steady electric current density $J$, and is subjected to a constant cross-sectional pressure $p$ by means of a spring pushing against the brush top as shown in figure 2 . The brush base of area $S$ continuously rests on the peripheral surface of a slip-ring/commutator of radius $R$ that rotates at a rather steady angular velocity $\Omega$. During operation, the brush continuously generates dissipative heat that originates at the interface between the stationary brush base surface, and the moving slip-ring/commutator peripheral surface due to: 1) friction caused by the actual sliding contact between the latter surfaces; 2) Joule's effect inherent to the electrical contact resistance and voltagedrop present at the interface; and, 3) mild ambient-induced corrosion acting exclusively at the interface also. The heat flux intrinsic to the latter dissipative processes $q_{O}$ is exclusively conducted away to the ambient through the brush base and body surfaces due to the high brush thermal conductance caused by the actual ambient gas forced convection. Joule's effect heat source inside the brush body may be ignored as compared to $q_{0}$, and heat loss at the brush top is negligible. However, the latter assumptions may be adequately relaxed if necessary at the expense of arriving at more complex formulations. Quasi-steady state heat conduction is assumed to be present in the brush in 
such way that the corresponding temperature distribution is one-dimensional only. The thermal conductivity of the brush $k$ is presumed to be constant over each time interval of interest, as well as $h_{a}$ that stands for the addition of appropriate averages of both the convective and linear radiative local heat-transfer coefficients between the brush body surfaces and the ambient. The actual magnitude of $q_{O}$, as set up by design and operation, is such that neither softening nor melting play any role in brush degradation; and, the brush wear is thought to be caused by mild corrosion and/or friction-induced erosion which are modelled as taking place exclusively at its base surface, assuming that the corrosion and/or erosion product is continuously and entirely washed away from the latter surface to be disseminated into the ambient. In this regard, a similar relaxation to that discussed in section 2.1 is also possible here, although in this case corrosion, at least, usually yields a nonsticking gaseous product. The temperature gradient at the brush base is such that thermal diffusion plays no role in the corrosion and/or erosion processes. The corrosion mechanisms are known, and their rates obey Arrhenius equation. The corrosion local heat generation rate is presumed to be sensibly constant over each time interval of interest also. Finally, figure 2 shows the brush base surface temperature $T_{o}$, as well as the average ambient temperature outside the thermal boundary layer $T_{a}$.

Then, a convenient relationship between $L$ and $T_{O}$, that is, the temperature which will be actually mapped by means of $I R$ thermography, may be reached at solving the heat conduction equation as applied to the brush under the above relevant assumptions [2]. In doing so, it is readily obtained that:

$$
\begin{gathered}
L=\frac{1}{m} \operatorname{Arth}\left[\frac{q_{o}}{k m\left(T_{o}-T_{a}\right)}\right) ; q_{o}=\zeta p \Omega R+\Sigma J+\gamma_{c b} \exp \left(\frac{-\alpha c b}{T_{o}}\right)+\gamma_{c s} \exp \left(\frac{-\alpha c s}{T_{o}}\right) \\
T(x)=T_{a}+\left(T_{o}-T_{a}\right) \frac{\cosh [m(L-x)]}{\cosh (m L)} \quad ; \quad 0 \leq x \leq L
\end{gathered}
$$

with:

$$
m=\sqrt{\frac{h_{a} P}{k S}} ; h_{a}=\Phi\left[\left\langle T(x)>, T_{a^{\prime}} a_{a^{\prime}} p_{a^{\prime}} \varepsilon_{o^{\prime}} P, L\right] ; k=\xi[\langle T(x)\rangle]\right.
$$

where $\zeta$ represents the friction coefficient between the brush base and the slip-ring/ commutator peripheral surface; $\Sigma$ accounts for an effective voltage-drop constant; $\alpha_{c b}, \gamma_{C b}$ and $\alpha_{c s}, \gamma_{c S}$ stand for characteristic parameters of the actual corrosion processes taking place at the brush base and the slip-ring/commutator peripheral surface respectively: $T(x)$ and $\langle T(x)\rangle$ mean the brush local temperature and its average respectively; $a_{a}$ and $p_{a}$ correspond to the average ambient gas flow rate and pressure outside the hydrodynamic boundary layer respectively; $\varepsilon_{o}$ denotes the brush body surface emissivity; and $P$ indicates the brush cross-sectional perimeter. The set of relations (5) through (7) constitutes the system of equations that represents the brush wear status at any time. It may be easily shown that a unique solution of this system is reached at if one IR thermographic mapping of $T_{o}$ is actually performed provided that $R, \varepsilon_{0}, p, J, \Omega, T_{a}, Q_{a}, p_{a}, S$ and $P$ are concurrently measured along this mapping by suitable means while $\zeta$ and $\Sigma$ are properly estimated from these measurements [2]. The latter solution contains $L$ that is used to characterize and diagnose the brush wear status.

\section{Bearing/electric brush wear evolution prediction}

Once the actual component wears have been obtained as shown in section 2, their subsequent evolution with time may be appraised, under given steady operating and ambient conditions for each time interval of interest, using time-dependent quantitative estimates of their respective wear rates. Indeed, the latter wear rate estimates may be coupled with their corresponding sets of solving thermal equations in section 2 (by means of appropriate iterative techniques) to actually yield reciprocal sets of component wear 
estimates that may be utilized in decision making for predictive maintenance if desired. Then, the main assumptions used for the estimation of the above wear rates are discussed next. The actual corrosion mechanisms are known, and their rates obey Arrhenius equation. Erosion is the result of a continuous removal of bonded atoms from their equilibrium sites at the solid surface being subject to a steady friction force field that makes them to become free atoms. This process is thought to be caused by the action of excited local solid vibration modes driven by the actual dissipative heat on the solid bonded atoms whose potential barrier has been lowered by the actual friction force field. This situation, in turn, may be viewed as being a generalized zero-order dissociation reaction between an ideal quantum gas of vibration modes, and bonded atoms so that the collision and Eyring's absolute-rate theories fully apply. Moreover, it may be proved that the erosion rate takes on an Arrhenius-like form which explicitly incorporates the friction force field effect $[2,4]$. Corrosion and erosion act independently from each other so that the component wear rate is obtained adding their respective rates. Thus, it may be shown that the application of the above assumptions yields the bearing wear rate and the brush wear rate as follows:

$$
\frac{\mathrm{d} \delta}{\mathrm{d} t}=\beta_{c a} \exp \left(\frac{-\alpha_{c a}}{T_{i}}\right)+\beta_{e a} \exp \left[\frac{-\alpha_{e a}}{T_{i}}\right) ; \frac{\mathrm{d} L}{\mathrm{~d} t}=-\beta_{c b} \exp \left(\frac{-\alpha_{c b}}{T_{o}}\right)-\beta_{e b} \exp \left(\frac{-\alpha_{e b}}{T_{o}}\right)
$$

where $\alpha_{c a}, \beta_{c a}$ and $\alpha_{e a}, \beta_{e a}$ stand for characteristic parameters of the actual corrosion and erosion processes taking place at the bearing annular surfaces respectively; $\alpha_{c b}, \beta_{c b}$ and $\alpha_{e b}, \beta_{e b}$ account for characteristic parameters of the actual corrosion and erosion processes taking place at the brush base surface respectively; and $t$ means time. Further, both $\alpha_{e a}$ and $\beta_{e a}$ depend on the bearing body material cohesive energy, $\delta, \mu$ and $\Omega$ whereas $\beta_{e a}$ alone depends on $T_{j}$. Likewise, both $\alpha_{e b}$ and $\beta_{e b}$ depend on the brush body material cohesive energy, $\zeta$ and $\rho$ whereas $\beta_{e b}$ alone depends on $T_{O}$.

\section{Bearinglelectric brush aging failure assessment}

The impact of the actual component wears on the potential for component failure due to aging as a function of time may be assessed, under given steady operating and ambient conditions for each time interval of interest, using time-dependent quantitative estimates of their respective failure rates. The latter failure rate estimates may be obtained by means of the load-strength interference theory of reliability coupled with the relevant sets of solving thermal equations in section 2 as follows. The latter theory allows the establishment of definite relationships between the mean values of the time-independent probability density functions that may properly represent both the actual loads and strengths acting on the component over each time interval of interest, and its respective failure rate at any moment [2,5]; whereas the latter thermal equations may be solved coupled with the wear rates discussed in section 3 yielding reasonable estimates of the latter mean values for each time interval of interest, using as initial condition of the solving procedure the actual component wear obtained by means of IR thermography as shown in section 2. In doing so, the time interval piece-wise scheme inherent to the solving procedure is suitably designed as to insure that all requirements implicit to the above method are duly fulfilled. In this regard, the use of time-independent probability density functions as indicated above yields by itself that component failure may occur in random fashion only over each time interval of interest. Moreover, if several component failure modes are feasible, they are assumed to be either independent or mutually exclusive so that the total component failure rate $\lambda$ may be obtained adding all the respective failure mode rates $[2,5]$; thus, $\lambda$ may be utilized in conjunction with reliability concepts to aid in decision making for preventive maintenance if desired [3]. The above method may be used, in particular, with time-independent exponential probability density functions as being representative of both the actual loads and strengths acting on the component yielding that failure rates are directly, and inversely proportional to their respective mean 
values provided that the ratio between the latter values is small enough as to insure high reliability for the component [2]. Then, this modality may be shown to be suitable for the estimation of the bearing aging failure rate; and it is applied next assuming that failure may occur due to either undue wear, or excessive stress that includes the effect of the actual local temperature distribution in (3). For the first mode, loads are represented by the actual wear rates, and strengths are taken as being proportional to the difference between the maximum allowable bearing annulus $\delta M$ and the actual $\delta$ 's; whereas for the second mode, loads are represented by the actual absolute maxima of the normal stress components $\sigma_{M}$ 's, and strengths are characterized by either the actual yield or rupture stresses $\sigma_{S}$ 's respectively. In doing so, $\sigma_{M}$ 's may be properly quantified assuming that: 1) the uncoupled quasi-static thermoelastic theory is applicable; 2) the bearing is axially unrestrained; and, 3 ) the bearing weight may be ignored. Further, closed-form expressions for the $\sigma_{M}$ 's may be obtained if the equivalent hydrostatic stress $\sigma(r)$ is used instead for characterizing the normal stress components [3]. Thus, it may be shown that the application of the preceding concepts finally yields $[2,3]$ :

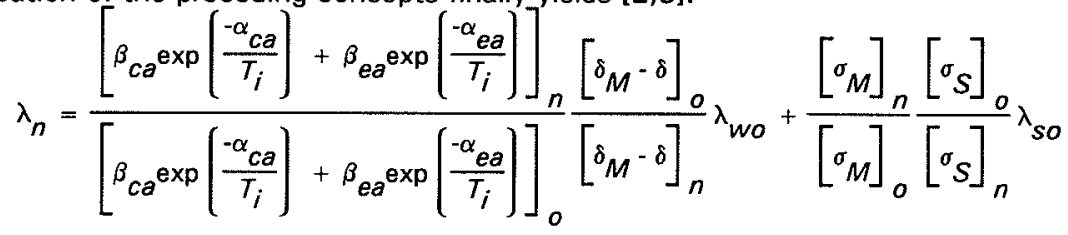

$$
\begin{aligned}
& n=1,2,3, \ldots \ldots
\end{aligned}
$$

with:

$$
\sigma_{M}=\sigma\left(R_{j}\right) \text { if } \sigma\left(R_{i}\right)+\sigma\left(R_{0}\right) \leq 0 \text { or } \sigma_{M}=\sigma\left(R_{0}\right) \text { if } \sigma\left(R_{j}\right)+\sigma\left(R_{0}\right) \geq 0
$$

and:

$$
\begin{gathered}
\sigma\left(R_{j}\right)=A\left[1+\frac{2 R_{o}^{2}}{R_{o}^{2}-R_{i}^{2}} \ln \left[\frac{R_{i}}{R_{o}}\right]\right]+B ; \quad \sigma\left(R_{o}\right)=A\left[1+\frac{2 R_{i}^{2}}{R_{o}^{2}-R_{i}^{2}} \ln \left[\frac{R_{i}}{R_{o}}\right]\right]+B \\
A=\frac{\alpha E}{3(1-\nu)} \frac{R_{o} h_{a}}{k}\left(T_{o}-T_{a}\right) ; \quad p_{i}=\eta\left(T_{i}, \chi\right) ; B=\frac{2 R_{o}^{2}}{3\left[R_{o}^{2}-R_{i}^{2}\right]}\left(p_{i}-p_{a}\right)-\frac{2}{3} p_{i}
\end{gathered}
$$

where $\lambda_{w o}, \lambda_{\text {so }}$ denote the wear and stress failure mode rates of a newly-installed bearing respectively; $n$, o refer to evaluation at the beginning of the $n$th time interval of the solving procedure and for a newly-installed bearing respectively; $\alpha, E$ and $\nu$ stand for the linear thermal expansion coefficient, the Young's modulus and the Poisson's ratio of the bearing body material respectively; and $p_{i}$ means the lubricating fluid pressure. The brush aging failure rate may be deduced in like manner though cannot be shown due to lack of space.

\section{REFERENCES}

[1] MADRID (A.). - Evaluation of aging in cracked/voided piping using IR thermography. 1992 ASNT Spring Conference Paper Summaries, Orlando, Florida, April 1992, p.154-156. [2] MADRID (A.). - Using IR thermography for quantifying failure rates of electric power network components. Infrared Technology XVI, I.J. Spiro, Editor, Proc. SPIE Vol.1341, San Diego, California, July 1990, p.24-45.

[3] MADRID (A.). - Infrared thermography as an aid in quantitative reliability and risk analyses. Applications of Infrared Technology, T.L. Williams, Editor, Proc. SPIE Vol.918, London, England, June 1988, p.40-60.

[4] YOURGRAU (W.), VAN DER MERWE (A.) and RAW (G.). - Treatise on irreversible and statistical thermophysics. Dover Publications, Inc., New York, New York, 1982, Chp.3-4.

[5] LEWIS (E.E.). - Introduction to reliability engineering. John Wiley \& Sons, Inc., New York, New York, 1987, Chp.4-6. 